\title{
Occlusive Thrombosis With Neovascularization of the Internal Carotid Artery
}

\author{
-Two Case Reports-
}

\author{
Kazumichi YoshIDA, Katsumi InOUE*, Masaki CHIN, \\ Osamu NARUMI, and Sen YAMAGATA \\ Departments of Neurosurgery and \\ ${ }^{*}$ Cardiology, Kurashiki Central Hospital, Kurashiki, Okayama
}

\begin{abstract}
Two patients presented with sudden onset of occlusive thrombosis of the atherosclerotic carotid artery. The patients underwent carotid endarterectomy. Histological examination of the specimens clearly showed the presence of pre-existing atherosclerotic plaque with moderate stenosis and occlusive organized thrombus. Blood flow was maintained through a honeycomb pattern of multiple neovascularization within the organized thrombus. These cases suggest that both the degree of stenosis and the plaque characteristics should be considered when evaluating the risk of stroke in patients with carotid artery stenosis.
\end{abstract}

Key words: atherosclerosis, vulnerable plaque, occlusive thrombosis, neovascularization

\section{Introduction}

The factors influencing the risk of stroke associated with low grade carotid artery stenosis remain to be elucidated. Vulnerable plaque in areas with moderate stenosis, rather than severe stenosis, may cause acute thrombotic occlusion in patients with coronary artery lesions, resulting in the so-called acute coronary syndrome. ${ }^{3,4,12)}$ In contrast, both severity of stenosis and plaque stability are extremely important in determining the risk of future stroke in patients with carotid atherosclerosis. The pathological mechanisms operating in coronary lesions may also occur in the carotid arteries. ${ }^{7)}$

Here we describe two cases of occlusive thrombosis in the atherosclerotic carotid artery with moderate stenosis and subsequent neovascular channel formation in the organized thrombus which were confirmed by histological examination.

\section{Case Reports}

Case 1: A 64-year-old woman receiving oral therapy for hyperlipidemia presented with amaurosis fugax affecting her left eye. She had no history of atrial fibrillation. Angiography showed a highly irregular stenosis of the left internal carotid artery (ICA).

Received April 6, 2007; Accepted June 14, 2008
Therefore, carotid endarterectomy (CEA) was planned. The region with the greatest degree of stenosis contained several fine, thread-like channels of blood flow, which merged into the peripheral ICA (Fig. 1).

Case 2: A 69-year-old woman receiving oral therapy for hyperlipidemia and diabetes mellitus had been regularly followed up at the hospital. She had no history of arrhythmia or heart disease. The patient underwent neurological evaluation after an episode of loss of consciousness. Angiography showed severe stenosis in the right ICA, as well as scant anterograde blood flow distal to the stenosis as far as the intracranial portion of the ICA, indicating a socalled pseudo-occlusive state (Fig. 2). Thus, CEA was performed.

\section{Histological Findings}

The lesions were excised en bloc without incision of the plaque interior during CEA. The specimens were transversely sectioned serially at 2-mm intervals and examined, particularly the portion with the highest degree of stenosis identified by carotid angiography. Gross examination found all specimens showed virtually total luminal occlusion (Figs. 3 and 4). However, microscopic neovascular channels were clearly observed (Figs. 5A, C and 6A, C). In Case 1, pre-existing fibrocalcific atherosclerotic plaque oc- 


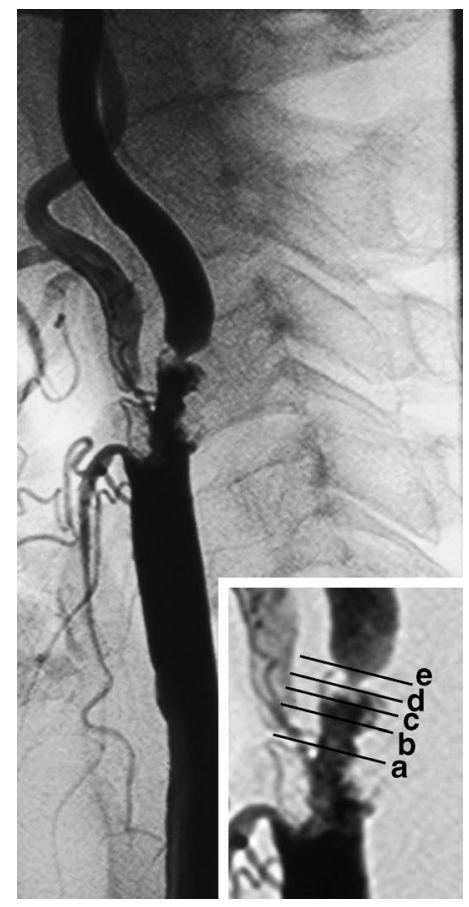

Fig. 1 Case 1. Left carotid angiogram (lateral projection, arterial phase) showing high grade stenosis with remarkably irregular lumen. Inset, magnified view of the carotid bifurcation showing the region with the greatest degree of stenosis contains several fine, thread-like channels of blood flow, which merge into the peripheral internal carotid artery. Lines (a) to (e) correspond to the cross sections of the excised plaque in Fig. 3.

cupied only a moderate $(<50 \%)$ part of the intimal vessel area (Fig. 3). The chronic total occlusive lesion, which was caused by thrombosis, consisted mainly of abundant collagen fibers with patchy calcification (Fig. 5B, D). Neovascular channels of various forms and sizes were clearly observed within the organized thrombus (Fig. 5B, D). Similarly in Case 2, organized occlusive thrombus with neovascularization was found within a moderate atherosclerotic stenotic carotid lesion. Smooth muscle cells bordering the new blood vessels, together with arterial changes in the new vessels, were also noted (Fig. 6B, D).

\section{Discussion}

The present cases of sudden severe stenosis of the carotid arteries were caused by occlusive thrombosis in the atherosclerotic carotid artery with moderate stenosis and subsequent neovascular channel formation in the organized thrombus, as histologi-

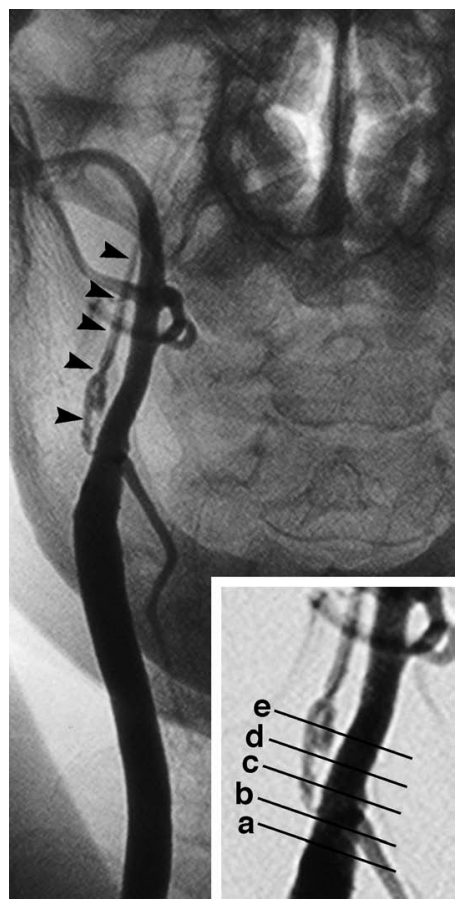

Fig. 2 Case 2. Right carotid angiogram (anteroposterior projection, arterial phase) demonstrating high grade stenosis with scant anterograde blood flow as far as the intracranial portion of the internal carotid artery, a so-called pseudo-occlusion (arrowheads). Inset, magnified view of the carotid bifurcation. Lines (a) to (e) correspond to the cross sections of the excised plaque in Fig. 4.

cally confirmed in the excised plaque obtained at CEA. The abrupt increase in the degree of stenosis in our patients could be explained by the presence of occlusive thrombosis arising from pre-existing plaque, caused either by disruption of the fibrous cap or development of vascular endothelial erosion. Thorough examination of emergency CEA specimens has clearly demonstrated the importance of intraplaque hemorrhage and associated intraluminal thrombosis in the development of complete carotid occlusion. ${ }^{1)}$ A postmortem study confirmed that almost half of patients with carotid thrombotic occlusion had only moderate narrowing of the lumen $(<60 \%) .{ }^{11)}$

Acute occlusive thrombosis of the carotid arteries may be either asymptomatic or present with only mild symptoms if the circle of Willis or other intracranial collateral circulation is adequate, as in our two cases. However, low grade carotid artery stenosis with vulnerable plaque may lead to acute thrombotic occlusion resulting in formidable stroke in patients with insufficient collateral blood flow. 


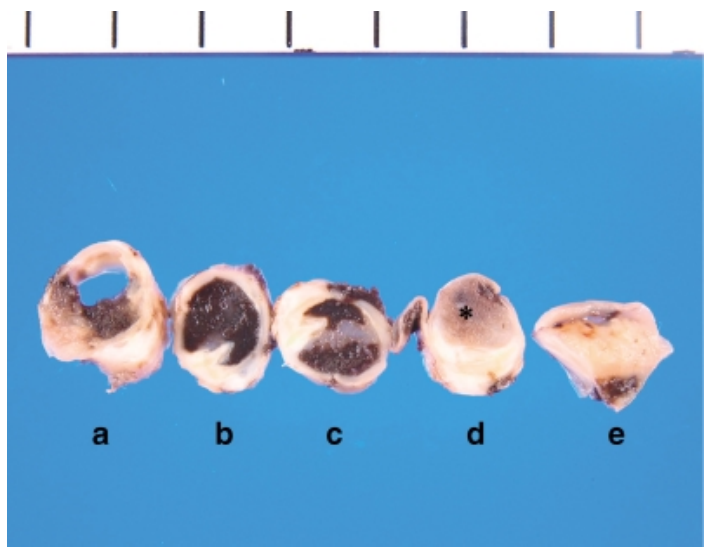

Fig. 3 Case 1. Photographs of the cross sections of excised plaque. Section (d) corresponding to the portion with the highest degree of stenosis reveals virtually total luminal occlusion with organized thrombus (asterisk). Pre-existing fibrocalcific atherosclerotic plaque occupied only moderate $(<50 \%)$ intimal vessel area.

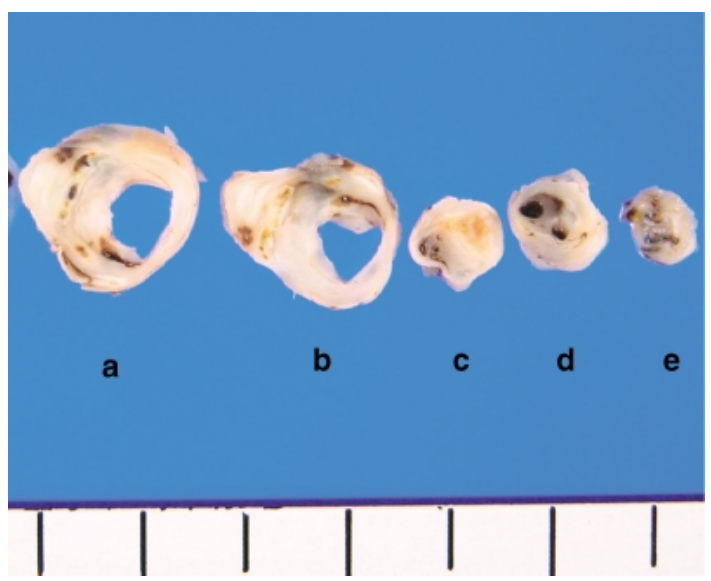

Fig. 4 Case 2. Photographs of the cross sections of excised plaque. Sections from (c) to (e) correspond to the portion with the highest degree of stenosis.

These findings suggest that both the degree of stenosis and the plaque characteristics should be considered when evaluating the risk of stroke in patients with carotid artery stenosis. ${ }^{6}$

Some cases of spontaneous recanalization after ICA occlusion have been confirmed by repeat angiography. ${ }^{2,5,8,9)}$ However, the timing and mechanism of spontaneous recanalization remains obscure. Various mechanisms have been proposed for spontaneous recanalization, including peripheral migration of occlusive clots, spontaneous clot lysis, and vasospasm..$^{2,5,8,9)}$ Neovascular channel formation has
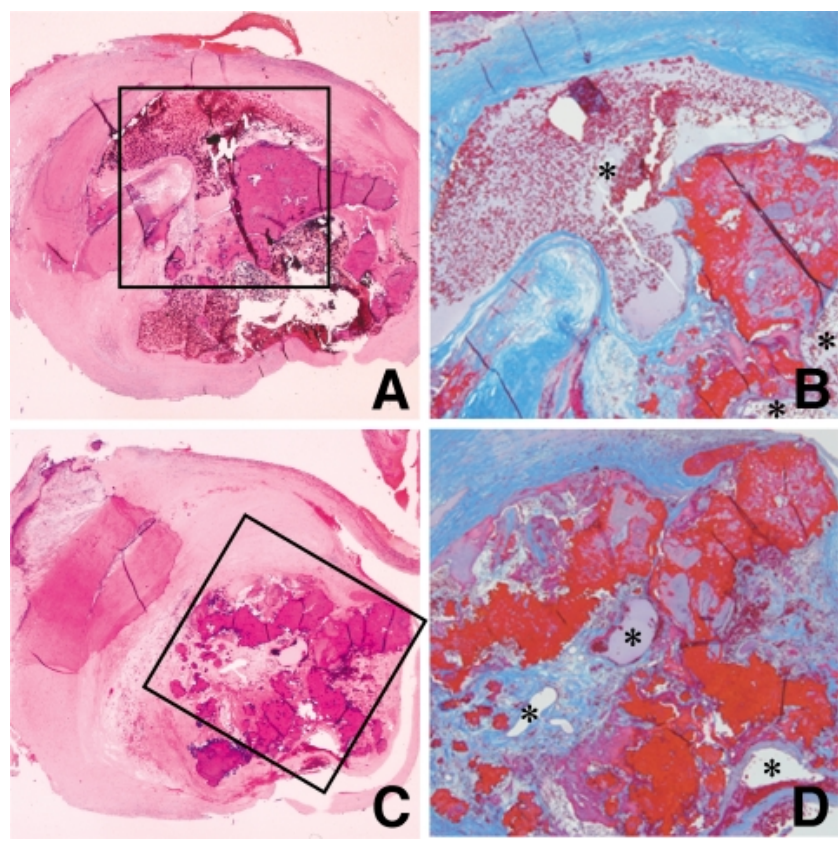

Fig. 5 Case 1. A: Photomicrograph corresponding to section (c) in Fig. 3 clearly showing the heterogeneous component of the occlusive lumen. Hematoxylin and eosin stain, original magnification $\times 6.55$. B: Photomicrograph of the area in the square in (A) demonstrating that the occluded lumen consists of abundant collagen fibers with organizing thrombus and patchy calcification. Neovascular channels with varied shapes and sizes (asterisks) are also clearly identified. Masson trichrome stain, original magnification $\times$ 16.4. C: Photomicrograph corresponding to section (d) in Fig. 3. Hematoxylin and eosin stain, original magnification $\times \mathbf{6 . 5 5}$. D: Photomicrograph of the area in the square in (C). Asterisks indicate neovascular channels. Masson trichrome stain, original magnification $\times 19$.

been observed in autopsy specimens of patients with chronic total coronary artery occlusion. ${ }^{10)}$ In the present two cases, close examination of the CEA specimens identified neovascularization in the organized thrombus, which could allow recanalization, although no angiographic evidence was available showing that the carotid artery had completely occluded and then later recanalized. Prospective studies of patients with moderate carotid artery stenosis and vulnerable plaque are needed to confirm this mechanism of recanalization with neovascular formation.

In the present two cases, histological examination revealed pre-existing atherosclerotic plaque with moderate stenosis, organized thrombus filling the 


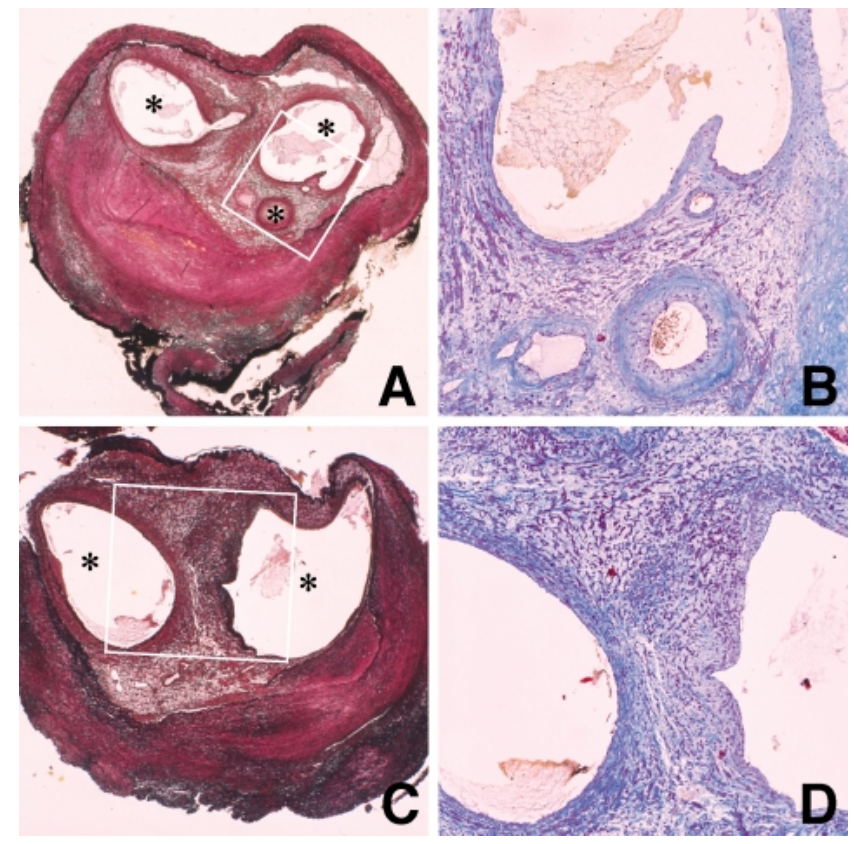

Fig. 6 Case 2. A: Photomicrograph corresponding to the section (d) in Fig. 4 revealing existence of an organized occlusive thrombus with neovascularization (asterisks) within a moderate atherosclerotic stenotic carotid lumen. Elastica van Gieson stain, original magnification $\times 6.55$. B: Photomicrograph of the area in the square in (A) demonstrating organizing thrombus consisting of abundant smooth muscle cells. Arterial changes of the new vessels are also observed. Masson trichrome stain, original magnification $\times 20$. C: Photomicrograph corresponding to the section (e) in Fig. 4. Asterisks indicate neovascular channels. Elastica van Gieson stain, original magnification $\times \mathbf{7 . 8 5}$. D: Photomicrograph of the area in the square in (C). Masson trichrome stain, original magnification $\times 16$.

vascular lumen, and blood flow via a honeycomb pattern of multiple micro-channels within the thrombus, which merged to maintain the blood flow distal to the site of occlusion. These histological findings indicate that considerable time had passed since the occlusive thrombosis formed, but the precise time frame is impossible to determine. Furthermore, in Case 2, a longer period of time had probably elapsed since thrombosis formation, as the histological findings included arterial changes in the new vessel wall of the organized thrombus.

\section{Acknowledgments}

We thank Ms. Kumi Nishida for excellent secretarial assistance and Ms. Kyomi Nakakuni for technical support in histological preparations.

\section{References}

1) Endo S, Hirashima Y, Kurimoto M, Kuwayama N, Nishijima M, Takaku A: Acute pathologic features with angiographic correlates of the nearly or completely occluded lesions of the cervical internal carotid artery. Surg Neurol 46: 222-228, 1996

2) Fieschi C, Bozzao L: Transient embolic occlusion of the middle cerebral and internal carotid arteries in cerebral apoplexy. J Neurol Neurosurg Psychiatry 32: 236-240, 1969

3) Fuster V, Badimon L, Badimon JJ, Chesebro JH: The pathogenesis of coronary artery disease and the acute coronary syndromes (1). N Engl J Med 326: 242-250, 1992

4) Fuster V, Badimon L, Badimon JJ, Chesebro JH: The pathogenesis of coronary artery disease and the acute coronary syndromes (2). N Engl J Med 326: 310-318, 1992

5) Irino $\mathrm{T}$, Taneda $\mathrm{M}$, Minami $\mathrm{T}$ : Angiographic manifestations in postrecanalized cerebral infarction. Neurology 27: 471-475, 1997

6) Kennedy J, Buchan AM: Acute neurovascular syndromes: hurry up, please, it's time. Stroke 35: 360-362, 2004

7) Lammie GA, Sandercock PA, Dennis MS: Recently occluded intracranial and extracranial carotid arteries. Relevance of the unstable atherosclerotic plaque. Stroke 30: 1319-1325, 1999

8) Markwalder TM, Starrett RW, Mumenthaler M: Spontaneous bilateral recanalization in bilateral internal carotid artery occlusion. Stroke 11: 95-98, 1980

9) Nguyen-Huynh MN, Lev MH, Rordorf G: Spontaneous recanalization of internal carotid artery occlusion. Stroke 34: 1032-1034, 2003

10) Srivatsa SS, Edwards WD, Boos CM, Grill DE, Sangiorgi GM, Garratt KN, Schwartz RS, Holmes DR Jr: Histologic correlates of angiographic chronic total coronary artery occlusions: influence of occlusion duration on neovascular channel patterns and intimal plaque composition. J Am Coll Cardiol 29: 955-963, 1997

11) Torvik A, Svindland A, Lindboe CF: Pathogenesis of carotid thrombosis. Stroke 20: 1477-1483, 1989

12) Virmani R, Burke AP, Farb A, Kolodgie FD: Pathology of the unstable plaque. Prog Cardiovasc Dis 44: 349-356, 2002

Address reprint requests to: Kazumichi Yoshida, M.D., Department of Neurosurgery, Kurashiki Central Hospital, 1-1-1 Miwa, Kurashiki, Okayama 710-8602, Japan. e-mail: ky7694@kchnet.or.jp 